\title{
Probabilidade de internação psiquiátrica e características sociodemográficas de portadores de depressão
}

\author{
The odds of psychiatric hospitalization and sociodemographic characteristics of people with depression
}

Probabilidad de internación psiquiátrica y características sociodemográficas de pacientes con depresión

Leonardo Naves dos Reis, Bruna Paiva do Carmo Mercedes² Adriana Inocenti Miasso ${ }^{3}$, Edilaine Cristina da Silva Gherardi-Donato ${ }^{4}$

\footnotetext{
${ }^{1}$ Enfermeiro, Mestre em Enfermagem Psiquiátrica. Discente do Programa de Pós-Graduação em Enfermagem Psiquiátrica, nível Doutorado, da Escola de Enfermagem de Ribeirão Preto (EERP) da Universidade de São Paulo (USP). Ribeirão Preto, SP, Brasil. E-mail: leonareis1@hotmail.com.

${ }^{2}$ Enfermeira. Discente do Programa de Pós-Graduação em Enfermagem Psiquiátrica, nível Mestrado, da EERP/USP. Ribeirão Preto, SP, Brasil. E-mail: bpaivadocarmo@yahoo.com.br.

${ }^{3}$ Enfermeira, Doutora em Enfermagem. Professora Doutora da EERP/USP. Ribeirão Preto, SP, Brasil. E-mail: amiasso@eerp.usp.br.

${ }^{4}$ Enfermeira, Doutora em Enfermagem Psiquiátrica. Professor Associado da EERP/USP. Ribeirão Preto, SP, Brasil. E-mail: nane@eerp.usp.br.
}

\section{RESUMO}

Este estudo objetivou verificar associação entre necessidade de internação psiquiátrica e características sociodemográficas dos usuários diagnosticados com depressão em um ambulatório de saúde mental. Realizou-se estatística descritiva e comparou-se o perfil dos depressivos com o perfil geral do serviço de saúde. Por meio da análise de regressão logística múltipla testou-se a associação da necessidade de internação com as variáveis independentes (sexo, idade e diagnóstico). Com relação aos pacientes depressivos, $82 \%$ são mulheres, 74\% possuem baixa escolaridade e $78 \%$ idade de 40 a 69 anos. Verificou-se associação da variável dependente com idade $(p=0,003)$ e depressão $(p=0,007)$; para a variável sexo não verificou-se associação. Tais resultados contribuem para a busca de estratégias de intervenção direcionadas às necessidades específicas desta clientela por parte da equipe ou ainda direcionando estudos que elucidem aspectos biopsicossociais relacionados ao perfil da clientela mais suscetivel, configurando-se como preâmbulo na construção do conhecimento para repensar as práticas de saúde mental. Descritores: Depressão; Epidemiologia; Saúde Mental; Enfermagem Psiquiátrica.

\section{ABSTRACT}

The objective was to verify the association between the need for psychiatric hospitalization and sociodemographic characteristics of mental health outpatients with depression. Descriptive statistics was performed. The patients' profiles were compared to the general health service clientele. Multiple logistics regression analysis was used to test the need for hospitalization considering independent variables (gender, ager and diagnosis). Regarding patients with depression, $82 \%$ were women, $74 \%$ had a low educational level, and $78 \%$ were 40 to 69 years old. An association was observed between the dependent variable and age $(p=0.003)$ and depression $(p=0.007)$; no association was observed for gender. These results contribute with the health team's search for intervention strategies aimed at the specific needs of this clientele and in guiding studies that elucidate the biopsychosocial aspects related to the profile of the most susceptible clientele, thus characterized as a first step to building the knowledge to rethinking mental health practices.

Descriptors: Depression; Epidemiology; Mental Health; Psychiatric Nursing.

\section{RESUMEN}

Se objetivó verificar asociación entre necesidad de internación psiquiátrica y características sociodemográficas de pacientes diagnosticados con depresión en ambulatorio de salud mental. Se aplicó estadística descriptiva, comparándose el perfil de depresivos con el perfil general del servicio. Mediante análisis de regresión logística múltiple, se testeó asociación de necesidad de internación con variables independientes (sexo, edad y diagnóstico). En relación a los pacientes depresivos, 82\% son mujeres, $74 \%$ con baja escolarización, $78 \%$ con entre 40 y 69 años. Se verificó asociación de la variable dependiente con edad $(p=0,003)$ y depresión ( $p=0,007)$; no se verificó asociación para variable sexo. Tales resultados contribuyen a la búsqueda de estrategias de intervención dirigidas a las necesidades específicas de estos pacientes por el equipo, inclusive orientando estudios que esclarezcan aspectos bio-psicosociales relacionados al perfil de los pacientes más susceptibles, configurándose como preámbulo en la construcción del conocimiento para repensar las prácticas de salud mental.

Descriptores: Depresión; Epidemiología; Salud Mental; Enfermería Psiquiátrica. 


\section{INTRODUÇÃO}

Estudos epidemiológicos sobre saúde mental podem mostrar o quanto as patologias psiquiátricas afetam a população mundial(1). Dados da Organização Mundial de Saúde apontam que uma em cada quatro pessoas no mundo irá experimentar uma condição de saúde mental ao longo da vida ${ }^{(2)}$. Os transtornos mentais compõem o quadro das doenças crônicas não transmissíveis e constituem um problema de saúde global e uma ameaça à saúde e ao desenvolvimento humano. A carga dessas doenças recai principalmente sobre países de baixa e média renda ${ }^{(1)}$.

Dentro dos dados epidemiológicos em saúde mental, os diversos transtornos têm contribuído para contextualizar esse panorama. Neste estudo, abordou-se como tema a depressão unipolar (DU), o qual tem sido foco de diversas pesquisas e discussões na atualidade inclusive na mídia ${ }^{(3)}$. A DU é considerada um importante problema em saúde mental e sua incidência é relativamente alta, tendo em vista que é comum e tende à remissão e recorrência. Atualmente a DU é a principal causa de incapacidade, quando medida pelo AVI (Anos Vividos com Incapacidade) e a segunda causa de AVAls (Anos de Vida Perdidos Ajustados com Incapacidade) entre as pessoas na faixa etária de 15 a 44 anos, para ambos os sexos (2).

De acordo com a Organização Mundial de Saúde a depressão é um transtorno mental comum que se apresenta com humor deprimido, perda de interesse ou prazer, sentimentos de culpa, diminuição da concentração e energia, baixa auto-estima e distúrbios do sono ou apetite. Este problema pode vir a se tornar crônico ou recorrente e resultar em deficiências substanciais na capacidade do indivíduo para cuidar de suas responsabilidades diárias( ${ }^{(2)}$. A depressão ainda pode apresentar graus (leve, moderado e grave) de intensidade diferentes de acordo com os sintomas apresentados, porém nos três graus o paciente apresenta um rebaixamento do humor, redução da energia e diminuição da atividade ${ }^{(4)}$.

A depressão acomete pessoas de todos os gêneros, idades e origens ${ }^{(5)}$. A literatura revela, todavia, que é mais frequente a ocorrência do primeiro episódio na adolescência e inicio da vida adulta, entre as mulheres, principalmente, devido as alterações hormonais ${ }^{(6)}$.
Em pesquisa recente, constatou-se que no Brasil, ao longo de 12 meses, os pacientes com faixa etária entre 50 e 64 anos de idade representaram 9,1\% daqueles com depressão. No mesmo estudo, esse acompanhamento também foi realizado na Espanha, sendo identificado que $5,4 \%$ dos pacientes nessa mesma faixa etária apresentavam depressão, porcentagem inferior à identificada no Brasil(5).

Após o advento da Constituição de 1988 e da lei $8.080 / 90$, a qual estipulou os princípios do Sistema Único de Saúde, vivenciamos um processo de transformações no modelo de assistência em saúde, inclusive no que se refere ao campo da saúde mental, através da compreensão da saúde-doença como um processo social, o que tem trazido a consequente proposição das práticas de desinstitucionalização(7).

Considerando-se os crescentes esforços voltados para a desinstitucionalização dos pacientes acometidos por transtornos psiquiátricos, assim como outros fatores como o aumento da expectativa de vida da população, é possivel inferir que tem ocorrido um aumento na demanda e na relevância dos serviços de níveis primário e secundário em saúde mental. Neste contexto de mudanças, se pode observar alguns desafios a serem enfrentados por estes serviços, para que possam se adequar ao novo panorama e atender de forma mais eficaz as necessidades de sua clientela.

Uma das principais lacunas está em conhecer a clientela atendida, conhecer o perfil dos usuários, buscando uma caracterização que possibilite direcionar melhor as ações dos serviços.

Dentre os princípios que norteiam o SUS destaca-se o da equidade, que visa reduzir as desigualdades, oferecendo tratamento diferente aos diferentes e o da integralidade que, entre outros aspectos, busca garantir o fornecimento de um conjunto articulado e contínuo de ações e serviços preventivos, curativos e coletivos em todos os níveis de assistência. O levantamento epidemiológico constitui importante ferramenta na manutenção destes princípios, fornecendo dados capazes de apontar especificidades do público atendido bem como explicitar suas reais necessidades de saúde ${ }^{(8)}$.

A importância dos estudos de cunho epidemiológico nos serviços de saúde se deve em grande parte, ao seu papel na produção de conhecimentos para a tomada de decisões no que se refere à formulação de políticas de 
saúde, à organização do sistema e às intervenções destinadas a dar solução a problemas específicos ${ }^{(9)}$.

Neste contexto de planejamento das atitudes em saúde mental, se torna necessário conhecer a demanda real que cada transtorno imprime ao serviço, considerando as peculiaridades de cada patologia, como o curso da doença, os prejuízos sociais da mesma e o tipo de tratamento estabelecido. Essa perspectiva permite melhorar a estrutura dos serviços em termos de recursos físicos e humanos.

O levantamento epidemiológico compõe um essencial instrumento orientador do planejamento e realização das atitudes em saúde, motivo este pelo qual o Ministério da Saúde denomina os sistemas de informações, tais como SIAB (Sistemas de Informação da Atenção Básica) e SIA (Sistema de Informações Ambulatoriais), como instrumentos de gestão, tamanha a importância conferida ao conhecimento epidemiológico(10).

A necessidade de se prestar atendimento integral e equânime aos usuários de serviços de saúde mental; a carência de informações sobre o perfil epidemiológico da população atendida e principalmente a possibilidade de implementação de estratégias que contribuam para prevenção de agravos em pacientes com diagnóstico de depressão, motivaram a realização deste trabalho com o objetivo de conhecer as características sociodemográficas destes pacientes em comparação à população geral atendida em um Núcleo de Saúde Mental, bem como a relação destas com a necessidade de internação psiquiátrica.

\section{MÉTODOS}

Estudo quantitativo descritivo e exploratório, de natureza epidemiológica, de prevalência e correlacional. A amostra foi composta por todos os indivíduos em tratamento no Núcleo de Saúde Mental (NSM) do Centro de Saúde Escola da Faculdade de Medicina de Ribeirão Preto, no período da coleta de dados, nos meses de abril e maio de 2012. As informações foram extraídas dos prontuários dos pacientes classificados como ativos no cadastro do serviço. O NSM é um serviço de natureza secundária e disponibiliza atendimento ambulatorial em saúde mental.

Na primeira etapa de análise dos dados, realizou-se a estatística descritiva das características sociodemográficas da amostra de pacientes, sendo consideradas as variáveis idade, em anos completos; sexo, dividido em masculino e feminino; diagnóstico, divididos conforme o capítulo $\mathrm{V}$ da CID-10 e nível de escolaridade, divididos nas categorias não alfabetizado, ensino fundamental incompleto, ensino fundamental, ensino médio incompleto, ensino médio, superior incompleto e superior. Descreveu-se ainda a variável denominada "internação", dividida em "sim", se o paciente já foi internado em unidade psiquiátrica pelo menos uma vez e "não", caso nunca tenha sido submetido a internação. Ainda nesta mesma etapa, realizou-se a comparação das características dos pacientes diagnosticados com depressão e o perfil geral do NSM.

Sabe-se que o diagnóstico psiquiátrico depende de diversos fatores, entre os quais a forma de interpretação do profissional médico, e levando-se em conta que o atendimento no NSM é realizado por três psiquiatras diferentes, sendo que cerca de $93 \%$ dos pacientes são divididos entre eles e os aproximadamente $7 \%$ restantes são atendidos por médicos residentes, admitiu-se um viés relacionado à variável "diagnóstico" sendo aceito o diagnóstico mais recente constante no prontuário do paciente, independentemente do profissional responsável pelo seu tratamento ${ }^{(11)}$.

Na segunda etapa da análise de dados foi testada a associação entre a variável dependente "internação" e as variáveis independentes (sexo, idade e diagnóstico). Para tanto, utilizou-se da análise de regressão logística com variáveis múltiplas e processo de seleção stepwise backward ${ }^{12)}$.

Após a análise de regressão, utilizou-se os coeficientes extraídos desta e relativos a cada uma das variáveis independentes que se adequaram ao modelo $(p<0,05)$ e elaborou-se a função logística da amostra do NSM (Figura 1) a qual possui como variável resposta a probabilidade de um paciente do NSM necessitar de internação psiquiátrica ao menos uma vez ao longo da vida. A mesma equação pode ser aplicada a pacientes novos no serviço como forma de estimativa da probabilidade de internação(12). 
Figura 1: Função logística (NSM)

$$
P(\mathrm{I})=\frac{1}{1+e^{-\left(\alpha+\Sigma \beta_{\mathrm{i}} X_{\mathrm{i}}\right)}}
$$

$P(I)=$ Probabilidade de um paciente do NSM necessitar de pelo menos uma internação ao longo da vida.

$a=$ parâmetro constante estimado com base nos dados amostrais.

$\beta=$ parâmetro constante estimado com base nos dados amostrais, no entanto, haverá um $\beta$ diferente para cada uma das variáveis dependentes.

Uma vez elaborada a equação, obteve-se as retas relativas a cada transtorno, em função da idade e probabilidade de internação ${ }^{(12)}$.

A pesquisa foi apresentada à equipe profissional do NSM e autorizada pelo responsável. Foi aprovada pelo Comitê de Ética em Pesquisa da Escola de Enfermagem de Ribeirão Preto da Universidade de São Paulo (protocolo 1446/2011), atendendo às normas estabelecidas pela Resolução 196/96 do Conselho Nacional de Saúde. Foi dispensado o uso do Termo de Consentimento Livre e Esclarecido (TCLE) devido à natureza secundária dos dados.

\section{RESULTADOS}

O senso realizado no NSM verificou que, na ocasião da coleta de dados, havia 1.281 pacientes em tratamento no referido serviço de saúde. Destes, 287 (cerca de 22\%), possuem depressão como diagnóstico principal, sendo o transtorno mais frequentemente verificado entre os pacientes do NSM.

Dentre os pacientes diagnosticados com depressão, quase $74 \%$ possuíam no máximo o ensino fundamental, enquanto que para o perfil geral de pacientes do NSM verificou-se que quase $67 \%$ apresentavam este mesmo nível de escolaridade. Já em relação aos níveis intermediários de escolaridade, verificou-se o oposto, sendo que entre aqueles que possuem diagnóstico de depressão o percentual encontrado foi de aproximadamente $20 \%$ e no perfil geral do NSM mais de $27 \%$.
Em se tratando dos níveis mais elevados de instrução, a realidade se inverte novamente, na qual o perfil geral do NSM apresenta cerca de um ponto percentual abaixo dos pacientes com depressão (Tabela 1). Ainda com relação aos níveis superiores de escolaridade, chama atenção o fato de que dentre todos os pacientes em tratamento no NSM, apenas 25 apresentam nível superior, no entanto, $36 \%$ destes estão entre os diagnosticados com depressão. 
Tabela 1: Comparações entre as distribuições por idade, gênero e nível de escolaridade dos pacientes diagnosticados com Depressão e quadro geral de pacientes no NSM. Ribeirão Preto, SP, 2012.

\begin{tabular}{|c|c|c|c|c|c|c|c|c|}
\hline \multirow{2}{*}{ Variável } & \multicolumn{4}{|c|}{ Depressão } & \multicolumn{4}{|c|}{ Total NSM } \\
\hline & $\mathbf{N}$ & $\%$ & Média & DP & $\mathbf{N}$ & $\%$ & Média & DP \\
\hline Idade (anos) & 375 & - & 52,79 & 12,45 & 1281 & - & 49,69 & 14,03 \\
\hline $18-29$ & 11 & 2,93 & & & 108 & 8,43 & & \\
\hline $30-39$ & 27 & 7,20 & & & 210 & 16,39 & & \\
\hline $40-49$ & 85 & 22,67 & & & 304 & 23,73 & & \\
\hline $50-59$ & 112 & 29,87 & & & 338 & 26,39 & & \\
\hline $60-69$ & 95 & 25,33 & & & 221 & 17,25 & & \\
\hline $70-79$ & 41 & 10,93 & & & 83 & 6,48 & & \\
\hline$\geq 80$ & 4 & 1,07 & & & 16 & 1,25 & & \\
\hline Gênero & 375 & & & & 1281 & & & \\
\hline Feminino & 307 & 81,87 & & & 880 & 68,70 & & \\
\hline Masculino & 68 & 18,13 & & & 401 & 31,30 & & \\
\hline Escolaridade & 171 & & & & 537 & & & \\
\hline Fundamental & 126 & 73,68 & & & 359 & 66,85 & & \\
\hline Médio & 34 & 19,88 & & & 147 & 27,37 & & \\
\hline Superior & 11 & 6,43 & & & 31 & 5,77 & & \\
\hline
\end{tabular}

No que se refere à distribuição por gênero, verificouse em relação à amostra geral de pacientes do NSM que as mulheres são a grande maioria, representando cerca de $69 \%$ do total. Contudo, entre os pacientes com depressão, esta diferença é ainda maior, sendo que as mulheres totalizam aproximadamente $82 \%$ (Tabela 1 ).

A comparação das distribuições etárias da amostra revelou que cerca de $78 \%$ dos indivíduos com depressão estavam na faixa de 40 a 69 anos. Já em relação ao perfil geral do NSM, observou-se que a faixa etária predominante foi a mesma verificada para os depressivos, no entanto com percentual menor (cerca de 67\%) (Tabela 1). A pirâmide etária referente aos pacientes depressivos apresenta deslocamento à direta em relação à pirâmide etária da população do NSM, demonstrando que, de uma forma geral, os pacientes com depressão são mais velhos. Outro dado que reflete esta realidade é a média de idade, sendo que na amostra geral do NSM verificou-se idade média de 49, 69 anos e entre os diagnosticados com depressão, 52, 79 anos.

Dentre os 1.281 pacientes em tratamento no NSM, pouco mais de $18 \%$ já foram internados em unidade psiquiátrica ao menos uma vez. Destes, apenas 14 (quase 6\%) possuem depressão como diagnóstico principal. Os 14 pacientes depressivos que já necessitaram de internação representam menos de $5 \%$ do total de pacientes com este diagnóstico.

Por meio da análise de regressão logística com múltiplas variáveis, observou-se que havia associação entre a variável dependente "internação" e a variável independente "idade" ( $p=0,003)$, diferentemente do que observou-se em relação à variável "sexo" ( $p>0,05)$. Verificou-se ainda associação entre a variável "internação" e alguns transtornos psiquiátricos, dentre eles depressão $(p=0,007)$.

Em seguida, elaborou-se a função logística referente à amostra do NSM e esta continha os coeficientes relativos à idade, e alguns tipos de transtornos (somente aqueles que se adaptaram ao modelo de regressão), fatores estes, sobre os quais foi verificada associação com a necessidade de internação (nível terciário de atenção) ao longo da vida, de acordo com a idade, e o transtorno diagnosticado (Gráfico 1). 
Gráfico 1: Probabilidade (\%) de um paciente do NSM, necessitar ou já ter necessitado de pelo menos uma internação ao longo da vida, segundo idade e transtorno mental. Ribeirão Preto, 2012.

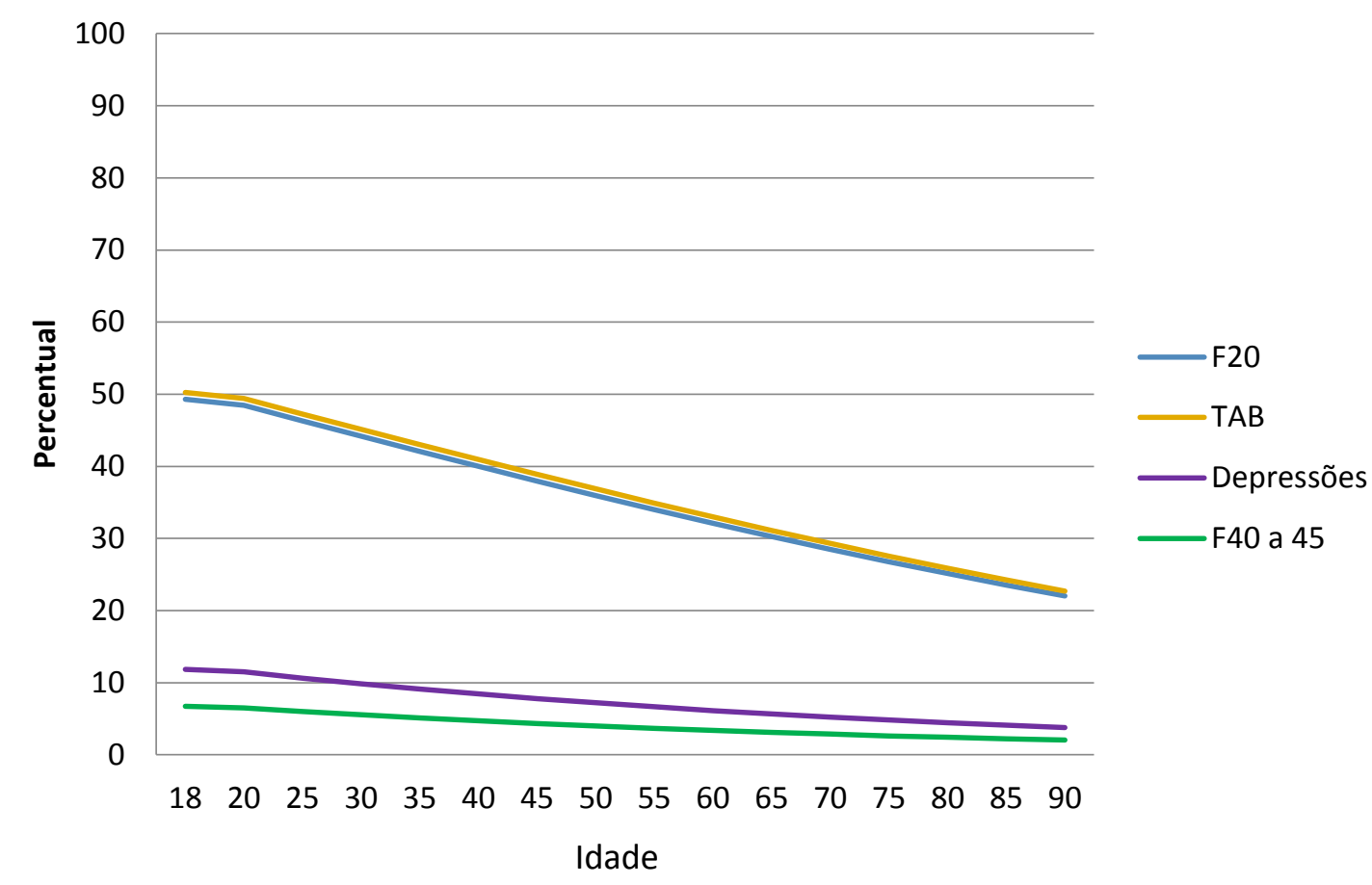

Verifica-se através do Gráfico 1, que a probabilidade de internação é inversamente proporcional à idade do paciente. O mesmo gráfico, exibe ainda outros três transtornos para efeito de comparação e evidencia que os pacientes diagnosticados com depressão apresentam baixa probabilidade de internação, se comparados com outros transtornos como esquizofrenia e transtorno afetivo bipolar. Por exemplo, um indivíduo com 35 anos de idade e diagnosticado com depressão possui pouco mais de $9 \%$ de chances de necessitar de internação pelo menos uma vez ao longo da vida. Já um paciente com esquizofrenia, com a mesma idade, apresenta aproximadamente $42 \%$ de chances.

\section{DISCUSSÃO}

Assim como verificado no NSM, observou-se em um ambulatório de saúde mental, situado na cidade de Maringá/PR, que a depressão constitui o transtorno mais frequentemente verificado, no entanto, para o referido estudo os pacientes depressivos representavam $29 \%$ do total e no NSM $22 \%{ }^{(10)}$. No Brasil, em um estudo que buscou avaliar os transtornos mentais em pacientes que procuraram atendimento em uma unidade de referência do Programa de Saúde da Família (PSF), a depressão foi o transtorno mais frequente, com uma prevalência de $17,6 \%{ }^{(13)}$. Tais estudos mencionados, assim como este, demonstram que a depressão constitui o transtorno mental mais frequente entre os usuários dos serviços de atenção primária e secundária.

Em estudo que avaliou o impacto dos sintomas depressivos na qualidade de vida dos usuários da rede básica de saúde, constatou-se que $72 \%$ dos pacientes tinham até o ensino fundamental, percentual muito próximo daquele verificado no NSM (cerca de $74 \%$ ), o que reforça a evidênca de que a maioria destes pacientes apresentam baixo nível de escolaridade ${ }^{(14)}$.

Resultados de estudos epidemiológicos desenvolvidos em diferentes nações, culturas e etnias, revelam que a depressão é cerca de duas vezes mais prevalente em mulheres do que em homens(15); o que também é verificado entre idosos, sendo que entre estes, os sujeitos de gênero feminino apresentam maior percentual de depressão(16). Mulheres apresentaram probabilidade cerca de três vezes maior de desenvolver depressão quando comparadas aos homens e dentre as possíveis explicações para isso, destacam-se as alterações hormonais nas diferentes fases da vida reprodutiva da mulher ${ }^{(6)}$.

Como se pode observar, no NSM, cerca de $67 \%$ dos pacientes com depressão possuem idade acima de 50 anos reforçando o que se verifica na literatura, que a depressão, de uma forma geral, atinge indivíduos mais 
velhos $^{(17)}$. Este achado é relevante para a prática clínica, pois apesar de no idoso os quadros depressivos apresentarem pior diagnóstico e maior incidência de suicídio, frequentemente são pouco valorizados pelos profissionais da saúde. Aponta ainda para a necessidade de interveções precoces e efetivas nessa população, visando um envelhecimento saudável(18-20).

A depressão, a ponto de ocasionar a necessidade de hospitalização do paciente, em geral, ocorre apenas em suas formas mais graves, nas quais o funcionamento do indivíduo torna-se bastante prejudicado, podendo haver inclusive risco de suicídio. Já nas formas mais "brandas" da doença, normalmente o tratamento em nível básico ou secundário é suficiente ${ }^{(2)}$. Isto talvez explique o fato de a depressão constituir o transtorno mais prevalente entre os pacientes do NSM e ainda assim ser uma das patologias que apresentam menor probabilidade de internação (Gráfico 1). Tal aspecto leva à reflexão sobre a possibilidade de parte destes pacientes serem atendidos na atenção primária, diminuindo a demanda do serviço especializado. Nesse contexto, o apoio matricial pode ser importante estratégia para a integração da equipe de saúde mental à equipe da Estratégia de Saúde da Família viabilizando ainda, a articulação da rede de serviços ${ }^{(18)}$.

\section{CONCLUSÃO}

Ao que se pôde observar, a depressão provavelmente constitui o transtorno mais prevalente entre os pacientes atendidos em serviços de atenção secundária à saúde

\section{REFERÊNCIAS}

1. Reis LN, Pereira SS, Cardoso L, Gherardi-Donato ECS. Transtornos Mentais Orgânicos em um Ambulatório de Saúde Mental Brasileiro. Revista Portuguesa de Enfermagem de Saúde Mental [Internet]. 2013 [acesso em: 20 dez 2013];(9):48-53. Disponível em:

http://www.scielo.gpeari.mctes.pt/pdf/rpesm/n9/n9a08.pdf. 2. World Health Organization. Burden of disease: DALYs - Part 4 [Internet]. In: World Health Organization. The global burden of disease: 2004 update. Geneva: World Health Organization; 2008 [acesso em: 20 dez 2013]. Disponível em:

http://www.who.int/healthinfo/global burden disease/GBD re port 2004update_part4.pdf.

3. Soares GB, Caponi S. Depressão em pauta: um estudo sobre o discurso da mídia no processo de medicalização da vida. Interface (Botucatu) [Internet]. 2011 [acesso em: $20 \mathrm{dez}$ 2013];15(37):437-46. Disponível em: http://dx.doi.org/10.1590/S1414-32832011005000006. 4. Organização Mundial de Saúde: Classificação Estatística Internacional de Doenças e Problemas Relacionados à Saúde CID-10 [Internet]. Genebra: Organização Mundial de Saúde; mental, além de atingir principalmente populações mais velhas, com baixo nível de escolaridade e mulheres. Tais informações denotam a necessidade de ações específicas, tanto direcionadas aos pacientes acometidos por este transtorno quanto preventivas no âmbito da atenção básica direcionadas aos grupos de risco.

Com relação à prevalência aumentada da depressão na população idosa, há de se levar em conta, o aumento desta população no Brasil e no mundo e principalmente o impacto da doença sobre estes indivíduos.

Os dados demonstrados neste estudo, também possuem importância relacionada ao princípio do SUS da equidade, de forma a dar tratamento peculiar aos depressivos, de acordo com as especificidades deste transtorno. A importância das informações aqui demonstradas também reside na necessidade de os serviços de atenção à saúde mental, neste caso relacionado aos pacientes com depressão, compreenderem o perfil de seus clientes, buscando estratégias de intervenção direcionadas às necessidades específicas deste grupo de pacientes, tornando possível a otimização do trabalho da equipe, da aplicação dos recursos e do atendimento integral.

Por fim, os resultados do presente estudo contribuem no direcionamento de outros estudos que possam elucidar aspectos biopsicossociais relacionados ao perfil da clientela mais suscetível, configurando-se como preâmbulo na construção do conhecimento científico para repensar as práticas de saúde mental.

2008 [acesso em: 20 dez 2013]. Disponível em: http://www.datasus.gov.br/cid10/V2008/cid10.htm. 5. Kessler RC, Birnbaum HG, Shahly V, Bromet E, Hwang I, McLaughlin KA, et al. Age differences in the prevalence and comorbidity of DSM-IV major depressive episodes: results from the WHO World Mental Health Survey Initiative. Depress Anxiety [Internet]. 2010 [acesso em: 20 dez 2013];27(4):351-64. Disponível em: http://dx.doi.org/10.1002/da.20634. 6. Lopez MRA, Ribeiro JP, Ores LC, Jansen K, Souza LDM, Pinheiro RT, et al. Depressão e qualidade de vida em jovens de 18 a 24 anos no sul do Brasil. Rev. psiquiatr. Rio Gd. Sul [Internet]. 2011 [acesso em: $20 \mathrm{dez} 2013$ ];33(2):103-8. Disponivel em: http://dx.doi.org/10.1590/S010181082011005000001.

7. Liberato MDM. Desinstitucionalizar é ultrapassar fronteiras sanitárias: o desafio da intersetorialidade e do trabalho em rede. Cadernos Brasileiros de Saúde Mental [Internet]. 2009 [acesso em: 20 dez 2013];1(1). Disponível em: http://www.incubadora.ufsc.br/index.php/cbsm/article/view/10 $\underline{16}$.

8. Malta DC, Castro AM, Gosch CS, Cruz DKA, Bressan A, Nogueira JD, et al. A política Nacional de promoção da saúde e a 
agenda da atividade física no contexto do SUS. Epidemiol. Serv. Saúde [Internet]. 2009 [acesso em: 20 dez 2013];18(1):79-86. Disponível em:

http://scielo.iec.pa.gov.br/pdf/ess/v18n1/v18n1a08.pdf. 9. Werneck G. Epidemiologia Descritiva: qualidade das informações e pesquisa nos serviços de saúde. Epidemiol. Serv. Saúde [Internet]. 2009 [acesso em: 20 dez 2013];18(3):205-7. Disponível em:

http://scielo.iec.pa.gov.br/pdf/ess/v18n3/v18n3a02.pdf. 10. Almeida GCM, Ferreira MAF. Saúde bucal no contexto do Programa Saúde da Família: práticas de prevenção orientadas ao indivíduo e ao coletivo. Cad Saude Publica [Internet]. 2008 [acesso em: 20 dez 2013];24(9):2131-40. Disponível em: http://dx.doi.org/10.1590/S0102-311X2008000900019. 11. Bloc L, Moreira V. Sintoma e fenômeno na psicopatologia fenomenológica de Arthur Tatossian. Rev. latinoam. psicopatol. fundam. [Internet]. 2013 [acesso em: 20 des 2013];16(1):28-41. Disponivel em: http://dx.doi.org/10.1590/S1415$\underline{47142013000100003 .}$.

12. Pagano M, Gauvreau K. Princípios de Bioestatística. Tradução da $2^{\text {a }}$ edição norte americana. São Paulo: Editora Cengage Learning; 2010.

13. Gonçalves DM, Kapczinski F. Prevalência de transtornos mentais em indivíduos de uma unidade de referência para Programa Saúde da Família em Santa Cruz do Sul, Rio Grande do Sul, Brasil. Cad Saude Publica [Internet]. 2008 [acesso em: 20 dez 2013];24(9):2043-53. Disponível em: http://dx.doi.org/10.1590/S0102-311X2008000900010. 14. Corso AN, Costa LS, Fleck MPA, Heldt E. Impacto de sintomas depressivos na qualidade de vida de usuários da rede básica de saúde. Rev Gaucha Enferm [Internet]. 2009 [acesso em: 20 dez 2013];30(2):257-62. Disponível em: http://seer.ufrgs.br/RevistaGauchadeEnfermagem/article/view/ $\underline{7317 .}$.

15. Seedat S, Scott KM, Angermeyer MC, Berglund P, Bromet EJ, Brugha TS, et al. Cross-national associations between gender and mental disorders in the World Health Organization World Mental Health Surveys. Arch Gen Psychiatry [Internet]. 2009 [acesso em: 20 dez 2013];66(7):785-95. Disponível em: http://dx.doi.org/10.1001/archgenpsychiatry.2009.36. 16. Drago SMMA, Martins RML. A depressão no idoso. Millenium [Internet]. 2012 [acesso em: $20 \mathrm{dez} 2013$ ];(43):79-94. Disponível em: http://www.ipv.pt/millenium/Millenium43/5.pdf.

17. Benedetti TRB, Borges LJ, Petroski EL, Gonçalves LHT. Atividade física e estado de saúde mental de idosos. Rev Saude Publica [Internet]. 2008 [acesso em: $20 \mathrm{dez} 2013$ ];42(2):302-7. Disponivel em: http://dx.doi.org/10.1590/S0034$\underline{89102008005000007}$.

18. Farfel JM, Fernandez R. Depressão. In: Jacob Filho W. Terapêutica no idoso - Manual da Liga do Gamia. $2^{a}$ ed. Rio de Janeiro: Rubio; 2008. p. 77-98.

19. Onocko-Campos RT, Campos GWS, Ferrer AL, Corrêa CRS, Madureira PR, Gama CAP, et al. Evaluation of innovative strategies in the organization of Primary Health Care. Rev Saude Publica [Internet]. 2012 [acesso em: $20 \mathrm{dez} 2013$ ];46(1):43-50.

Disponível em: http://dx.doi.org/10.1590/S003489102011005000083.

20. Silva ER, Sousa ARP, Ferreira LB, Peixoto HM. Prevalence and factors associated with depression among institutionalized elderly individuals: nursing care support. Rev Esc Enferm USP [Internet]. 2012 [acesso em: $20 \mathrm{dez} 2013$ ];46(6):1387-93. Disponivel em: http://dx.doi.org/10.1590/S0080$\underline{62342012000600015}$. 